\title{
Variation in neurosurgical management of traumatic brain injury: a survey in 68 centers participating in the CENTER-TBI study
}

\author{
Thomas A. van Essen ${ }^{1,2} \cdot$ Hugo F. den Boogert ${ }^{3}$ Maryse C. Cnossen ${ }^{4} \cdot$ Godard C. W. de Ruiter $^{2}$. lain Haitsma ${ }^{5}$. \\ Suzanne Polinder ${ }^{4} \cdot$ Ewout W. Steyerberg ${ }^{4,6} \cdot$ David Menon $^{7} \cdot$ Andrew I. R. Maas $^{8} \cdot$ Hester F. Lingsma $^{4}$. \\ Wilco C. Peul ${ }^{1,2} \cdot$ on behalf of the CENTER-TBI Investigators and Participants
}

Received: 18 October 2018 / Accepted: 30 November 2018 / Published online: 19 December 2018

(C) The Author(s) 2018

\begin{abstract}
Background Neurosurgical management of traumatic brain injury (TBI) is challenging, with only low-quality evidence. We aimed to explore differences in neurosurgical strategies for TBI across Europe.

Methods A survey was sent to 68 centers participating in the Collaborative European Neurotrauma Effectiveness Research in Traumatic Brain Injury (CENTER-TBI) study. The questionnaire contained 21 questions, including the decision when to operate (or not) on traumatic acute subdural hematoma (ASDH) and intracerebral hematoma (ICH), and when to perform a decompressive craniectomy (DC) in raised intracranial pressure (ICP).

Results The survey was completed by 68 centers (100\%). On average, 10 neurosurgeons work in each trauma center. In all centers, a neurosurgeon was available within $30 \mathrm{~min}$. Forty percent of responders reported a thickness or volume threshold for evacuation of an ASDH. Most responders (78\%) decide on a primary DC in evacuating an ASDH during the operation, when swelling is present. For ICH, $3 \%$ would perform an evacuation directly to prevent secondary deterioration and $66 \%$ only in case of clinical deterioration. Most respondents (91\%) reported to consider a DC for refractory high ICP. The reported cut-off ICP for DC in refractory high ICP, however, differed: $60 \%$ uses $25 \mathrm{mmHg}, 18 \% 30 \mathrm{mmHg}$, and $17 \% 20 \mathrm{mmHg}$. Treatment strategies varied substantially between regions, specifically for the threshold for ASDH surgery and DC for refractory raised ICP. Also within center variation was present: $31 \%$ reported variation within the hospital for inserting an ICP monitor and $43 \%$ for evacuating mass lesions.
\end{abstract}

This article is part of the Topical Collection on Brain trauma

The CENTER-TBI Investigators and Participants and their affiliations are listed at the end of the manuscript in the Appendix.

Portions of this work were presented at the 13th Symposium of the International Neurotrauma Society, Toronto, Canada, 2018.

Electronic supplementary material The online version of this article (https://doi.org/10.1007/s00701-018-3761-z) contains supplementary material, which is available to authorized users.

Thomas A. van Essen

essen@lumc.nl

1 Department of Neurosurgery, Leiden University Medical Center, University Neurosurgical Center Holland (UNCH), Leiden, The Netherlands

2 Department of Neurosurgery, Haaglanden Medical Center, University Neurosurgical Center Holland (UNCH), The Hague, The Netherlands

3 Department of Neurosurgery, Radboud University Medical Center, Nijmegen, The Netherlands
4 Center for Medical Decision Sciences, Department of Public Health, Erasmus Medical Center, Rotterdam, The Netherlands

5 Department of Neurosurgery, Erasmus Medical Center, Rotterdam, The Netherlands

6 Department of Biomedical Data Sciences, Leiden University Medical Center, Leiden, The Netherlands

7 Division of Anaesthesia, Addenbrooke's Hospital, University of Cambridge, Cambridge, UK

8 Department of Neurosurgery, Antwerp University Hospital and University of Antwerp, Edegem, Belgium 
Conclusion Despite a homogeneous organization, considerable practice variation exists of neurosurgical strategies for TBI in Europe. These results provide an incentive for comparative effectiveness research to determine elements of effective neurosurgical care.

Keywords Traumatic brain injury $\cdot$ Neurosurgery $\cdot$ Practice variation $\cdot$ Acute subdural hematoma

Neurosurgical decision-making in patients with traumatic brain injury (TBI) is often challenging for several reasons. First, no two TBI patients are identical—clinical and radiological findings may differ greatly [26]. Second, there is no high-quality evidence to support the range of possible neurosurgical procedures in TBI. Indications for surgical management are summarized in the Brain Trauma Foundation guidelines, [5] but are merely based on retrospective studies of small groups of selected patients. These guidelines provide general advice on surgical indications for evacuation of acute epidural (EDH), acute subdural (ASDH), and contusions/ intracerebral hematomas (ICH) based on the size of the hematoma and midline shift. The guidance for decompressive surgery is even less clear. It is mostly performed to decrease raised intracranial pressure (ICP), either as a primary procedure in an acute setting, or as a secondary procedure to deal with diffuse edema or peri-contusional swelling. The guidelines state that this latter use of secondary decompression can reduce ICP, but does not necessarily improve outcome [6]. More fundamentally, the rationale for ICP monitoring has been challenged by the BEST TRIP randomized controlled trial (RCT), which found no benefit of a management protocol based on intracranial pressure monitoring, compared to one based on serial imaging and clinical examination. These results have generated doubts regarding ICP monitoring $[1,7$, $15,20,28]$. Overall, there is no clear consensus on the indications, extent, and timing of surgery [32].

This limited high-quality evidence for surgical management in TBI arises from a lack of RCTs, which may be difficult to conduct due to pragmatic, ethical, and methodological barriers [3]; however, observational studies to determine effectiveness are more prone for bias [2]. A promising alternative approach could be comparative effectiveness research (CER) [24, 33]. In this design, the heterogeneity and variability, that trouble RCTs in TBI, are accepted and exploited to study effectiveness of treatments as they occur in real-life practice. The current Collaborative European Neurotrauma Effectiveness Research in Traumatic Brain Injury (CENTER-TBI) study aims to use CER methodology to study treatment effectiveness of several neurosurgical interventions [25].

The aim of this study was to explore differences in neurosurgical strategies for TBI across Europe to provide a context for CENTER-TBI, an up-to-date insight into European neurosurgical management of TBI, and to identify naturally occurring variation between trauma centers in order to identify substrates for neurosurgical research questions that might be answered using CER in the study.

\section{Materials and methods}

This study was conducted within the setting of the international observational study CENTER-TBI [25]. Between 2014 and 2015 , all centers participating in the international multicenter observational study CENTER-TBI (www.CENTER-TBI.eu) were asked to complete a questionnaire on neurosurgical management of TBI (Supplementary file 1) [9]. The questionnaire was sent to 71 centers (Fig. 1), of which five centers dropped out and two joined in, resulting in 68 eligible centers from Austria $(n=2)$, Belgium $(n=4)$, Bosnia Herzegovina $(n=2)$, Denmark $(\mathrm{n}=2)$, Finland $(\mathrm{n}=2)$, France $(n=7)$, Germany $(\mathrm{n}=4)$, Hungary $(n=3)$, Israel $(\mathrm{n}=$ 2), Italy $(n=10)$, Latvia $(\mathrm{n}=3)$, Lithuania $(\mathrm{n}=2)$, Norway $(n=3)$, Romania $(n=1)$, Serbia $(n=1)$, Spain $(n=4)$, Sweden $(n=2)$, Switzerland $(n=1)$, The Netherlands $(n=6)$ , and The United Kingdom $(n=7)$.

\section{Questionnaire development and administration}

We developed a set of questionnaires based on available literature and experts to measure the structure and processes of TBI care in individual centers. Details regarding this process and the questionnaires used are described in a separate paper [9]. Pilot testing was undertaken in 16 of the participating centers and feedback was incorporated into the final design.

One of the questionnaires was on neurosurgical standard practice. This survey contained 21 questions which could broadly be divided into 3 categories: (1) center characteristics and internal structure; (2) general (neuro) surgical trauma care and processes; and (3) site specific neurosurgical management for treating ASDH, EDH, ICH, the use of DC, and policy with regard to orthopedic injuries in the context of patients who had suffered a TBI.

Questions either sought quantitative estimates of key metrics (e.g., annual surgical volume, staff size, ASDH thickness, or ICP thresholds for surgery) or attempted to elicit the "general policy" of the center. To capture the latter, these questions were formulated in two ways: respondents were asked to 


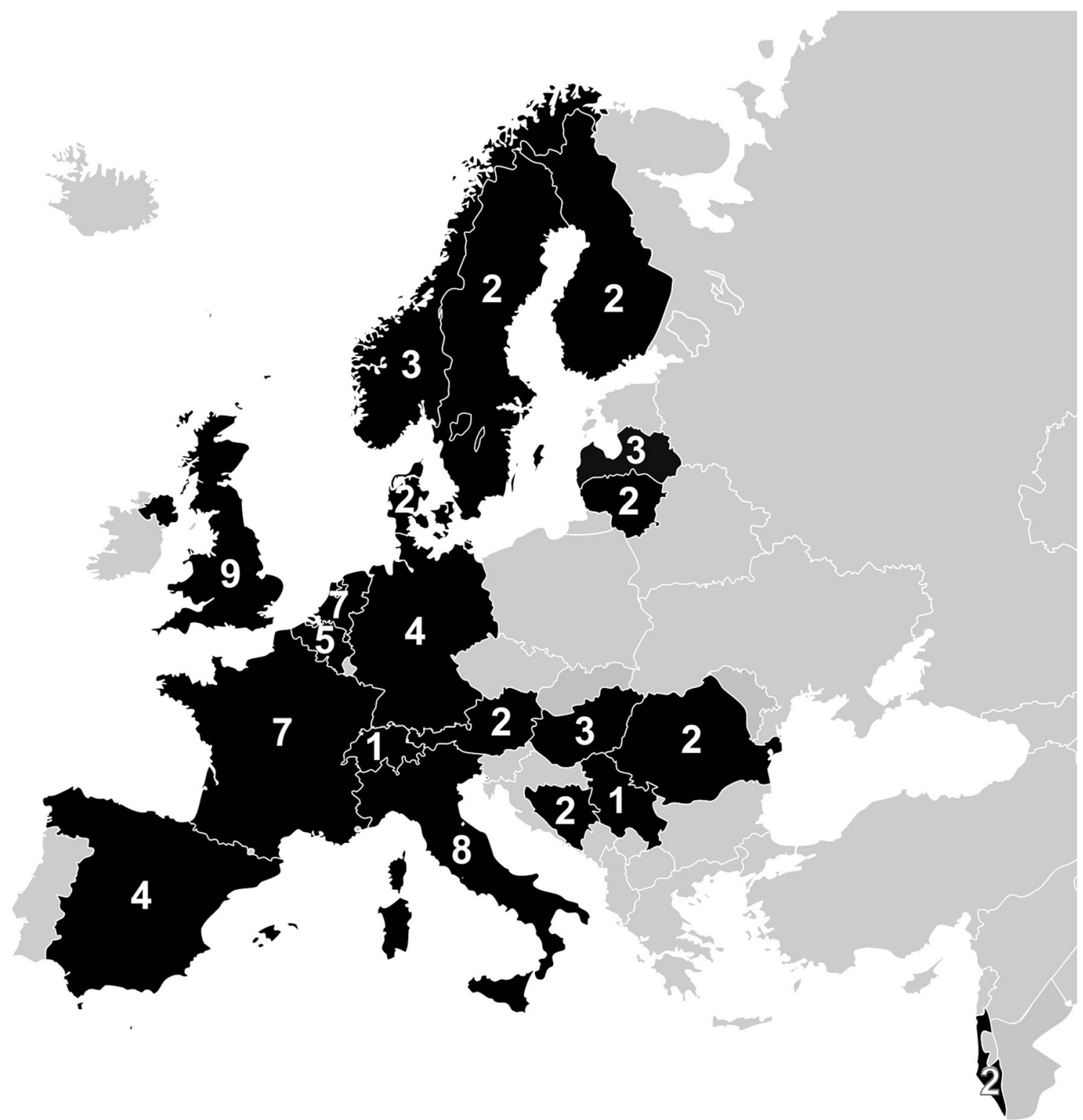

Fig. 1 Centers and countries included in the Collaborative European NeuroTrauma Effectiveness Research in Traumatic Brain Injury (CENTER-TBI) study. Reprinted and updated from Cnossen et al. (2016) with permission from Dr. Cnossen and Maas et al. (2015).

estimate what the management strategy is in more than three quarters of patients in their center in a given context; or respondents were asked to indicate how often they used a particular surgical technique or how often specific factors influence their decision-making (never $=0-10 \%$, rarely $=10-30 \%$, sometimes $=30-70 \%$, frequently $=70-90 \%$, and always $90-$ $100 \%)$. The options "frequently" and "always" were interpreted as "general policy", in line with a previous report [17] and similar to previous publications on other questionnaires $[8,9]$.

The reliability of the surveys was tested by calculation of concordance in a previous publication [9]. Overall, the median
Collaborative European NeuroTrauma Effectiveness Research in Traumatic Brain Injury: a prospective longitudinal observational study. Neurosurgery, 76:67-80, under a CC BY license, with permission from professor A.I. Maas

concordance rates between duplicate questions were 0.81 (range 0.44-0.97) and specifically for the "Neurosurgery" survey 0.78 (range $0.68-0.86$ ).

\section{Analyses}

The median and interquartile range (IQR) were calculated for continuous variables, and frequencies were reported along with percentages for categorical variables. Countries were divided into seven geographic regions: Northern Europe (Norway 3, Sweden 2, Finland 2 and Denmark 2 centers), Western Europe (Austria 2, Belgium 4, France 7, Germany 
4, Switzerland 1 and The Netherlands 6 centers), The United Kingdom (7 centers), Southern Europe (Italy 10 and Spain 4 centers), Eastern Europe (Hungary 3, Romania 1, Serbia 1 and Bosnia Herzegovina 2 centers), Baltic States (Latvia 3 and Lithuania 2 centers), and Israel ( 2 centers).

For the following neurosurgical treatment strategies, we quantified regional differences: an absolute cutoff of hematoma thickness as an indication for surgery for ASDH, DC in the primary evacuation of an ASDH, early/pre-emptive surgical evacuation for ICH, and DC as a general policy in case of refractory raised ICP.

To assess the association of region with one of these treatment choices, a logistic regression was performed with treatment choice (general policy or "yes/no") as a dependent variable and the region (categorical) as independent variable. Nagelkerke R2 indicated the variance explained by geographic region. Analyses were done in IBM SPSS Statistics version 20 (IBM, Chicago, IL, USA).

\section{Results}

\section{Center characteristics}

All 68 eligible centers completed the questionnaire on neurosurgery (response rate 100\%). Questionnaires were mainly completed by neurosurgeons ( $n=53,78 \%)$, followed by local CENTER-TBI investigators (mainly research physicians or nurses: 19\%). On average, 10 neurosurgeons (IQR 8-13) and four trauma surgeons (IQR 0-12) worked in each center. All centers reported that neurosurgical coverage was available $24 \mathrm{~h}$ a day/7 days a week, either by way of in-house availability of a qualified neurosurgeon (47\%), or the availability of such an individual in less than $30 \mathrm{~min}(53 \%)$ (Table 1).

\section{General (neuro) surgical care and processes}

Treatment decisions regarding cranial surgical interventions in TBI patients within the critical care ER and ICU period are in most centers determined by the neurosurgeon $(n=65,96 \%)$, followed by the orthopedic surgeons and neuro-intensivist in respectively $3 \%(n=2)$ and $1 \%(n=1)$. Urgent neurosurgical interventions (ICP monitor device insertion not included) for life-threatening traumatic intracranial lesions, are made by the neurosurgeon in $98.5 \%$ and trauma surgeons in $1.5 \%$ of the centers. Raised ICP will almost always be incorporated in decision-making, the time of day almost never (Fig. 2).

With regard to extremity fractures, the general policy in 59 (87\%) centers was so-called damage control with priority for TBI and delayed definitive treatment of the limb fractures (Table 2). This policy is protocolized in 21 centers (22\%).

Of all centers, $58(85 \%)$ estimated the space-occupying effect of traumatic lesions on the surrounding tissue by
Table 1 Characteristics of centers participating in neurosurgery survey

\begin{tabular}{|c|c|c|}
\hline Characteristic & $\begin{array}{l}N \\
\text { completed }\end{array}$ & $\begin{array}{l}\text { No. }(\%) \text { or } \\
\text { median } \\
(\mathrm{IQR})\end{array}$ \\
\hline Profession of respondent & 68 & \\
\hline Neurologist & & $3(4)$ \\
\hline Neurosurgeon & & $53(78)$ \\
\hline Trauma surgeon & & $3(4)$ \\
\hline ED physician & & $1(2)$ \\
\hline Intensivist & & $1(1)^{\mathrm{a}}$ \\
\hline Administrative staff member & & $11(16)^{\mathrm{a}}$ \\
\hline CENTER-TBI local investigator & & $13(19)^{\mathrm{a}}$ \\
\hline \multicolumn{3}{|l|}{ Volume of surgeries in $2013^{\mathrm{c}}$} \\
\hline $\mathrm{ASDH}$ & 59 & $25(15-49)$ \\
\hline $\mathrm{ICH} /$ contusion & 58 & $10(5-21)$ \\
\hline $\mathrm{EDH}$ & 59 & $10(5-19)$ \\
\hline \multicolumn{3}{|l|}{$\mathrm{DC}$} \\
\hline Hemicraniectomy & 57 & $10(5-16)$ \\
\hline Bifrontal & 57 & $0(0-2)$ \\
\hline Removal bone flap & 55 & $1(0-3)$ \\
\hline Ventriculostomy & 57 & $7(2-21)$ \\
\hline Cranioplasty & 56 & $10(6-14)$ \\
\hline Depressed skull fracture & 57 & $5(2-12)$ \\
\hline \multicolumn{3}{|l|}{ Staffing (FTE) } \\
\hline Neurosurgeons & 66 & $10(8-13)$ \\
\hline Residents in training & 65 & $5(3-8)$ \\
\hline Residents not in training & 61 & $0(0-3)$ \\
\hline Trauma surgeons & 64 & $4(0-12)$ \\
\hline \multicolumn{3}{|l|}{ Organization of care } \\
\hline $\begin{array}{l}\text { Neurosurgical decision making in } \\
\text { ICU }\end{array}$ & 68 & \\
\hline Neurosurgeon & & $65(96)$ \\
\hline Trauma surgeon & & $1(3)$ \\
\hline Neurologist & & 0 \\
\hline Neurointensivist or general intensivist & & $1(2)$ \\
\hline $24 / 7$ neurosurgical coverage ${ }^{b}$ & 68 & \\
\hline Qualified neurosurgeon in-house & & $32(47)$ \\
\hline Resident neurosurgery in-house & & $30(44)$ \\
\hline Neurosurgeon within $30 \mathrm{~min}$ & & $36(53)$ \\
\hline Neurosurgical resident within $30 \mathrm{~min}$ & & $11(16)$ \\
\hline Neurosurgeon more than $30 \mathrm{~min}$ & & $0(0)$ \\
\hline
\end{tabular}

$A S D H$ acute subdural hematoma, $E D H$ epidural hematoma, $I C H$ intracerebral hematoma, $D C$ decompressive craniectomy, FTE full time equivalent, $I C U$ intensive care unit

${ }^{\text {a }}$ Numbers do not add up because the local investigators also depicted their profession and one responder declared to be an intensivist as well as an administrative staff member

${ }^{\mathrm{b}}$ Multiple options possible

${ }^{\mathrm{c}}$ Head trauma-related surgeries 
calculation of the thickness of the hematoma and midline shift on CT. A quarter of centers used actual volume measurement to make surgical decisions (Table 2).

\section{Neurosurgical management of ASDH, EDH, ICH, and the use of decompressive craniectomy}

ASDH provided the highest volume of neurosurgical TBI cases, on average 25 cases per year. When performing a DC (for any indication), hemicraniectomy was the preferential technique, and bifrontal craniectomy was rarely performed (Table 1). Less than half of the centers $(n=27,40 \%)$ reported an absolute threshold for evacuating an ASDH. Four out of 10 centers generally incorporate age in their decision for evacuating an ASDH (Table 2 and Fig. 2).

ICH were seldom operated upon pre-emptively, but $67 \%$ of centers reported undertaking delayed surgery in the event of deterioration. Almost a third of centers reported within-center variations between individual neurosurgeons in decisions regarding surgical evacuation of contusions or traumatic ICH.

Only a very low proportion of centers would routinely perform a DC at the time of evacuation of either ASDH or ICH (respectively $6 \%$ and $1.5 \%$ of the centers). For refractory raised ICP, most centers $(n=64,91 \%)$ would consider a decompressive craniectomy, while $32(47 \%)$ see this as a general policy in their center (Fig. 3, Table 2 and figure in supplementary file 2). Ninety-six percent $(n=65)$ reported to have a specific threshold for DC in refractory raised ICP. This was most commonly specified as $25 \mathrm{mmHg}(n=39$, $58 \%)$, followed by $30 \mathrm{mmHg}(n=12,18 \%)$ and $20 \mathrm{mmHg}$ $(n=11,17 \%)$.

\section{Guidelines and practice variation}

Overall, the reported adherence to the BTF guidelines was high (Fig. 4). The use of surgical interventions and specific indications for these interventions varied substantially within and between regions (Table 3). Surgical evacuation of ICH was only performed in the Baltic States and Southern Europe and geographic region explained $35 \%$ of the variance in use of the intervention. Having a specific threshold for ASDH surgery and employing a DC for refractory-raised ICP showed the largest within-region and also betweenregion variation. Lastly, when directly asked whether variation in specific management strategies exist, respectively $31 \%$ and $43 \%$ indicated to have a structural variation within their center staff with regard to ICP sensor insertion and mass lesion evacuation (Table 4).

\section{Discussion}

The aim of this study was to explore differences in neurosurgical strategies for TBI across Europe. We found substantial variability in practice and thereby provide useful indications regarding potential substrates for CER in CENTER-TBI. The structures and processes of neurosurgical care are generally homogeneous across centers with a comparable number of neurosurgeons, similar organization of neurosurgical coverage and uniform organization of responsibility for most surgical decisions on the ER and ICU. The indications for surgery, however, differ substantially with high within-region and between-region practice variations.

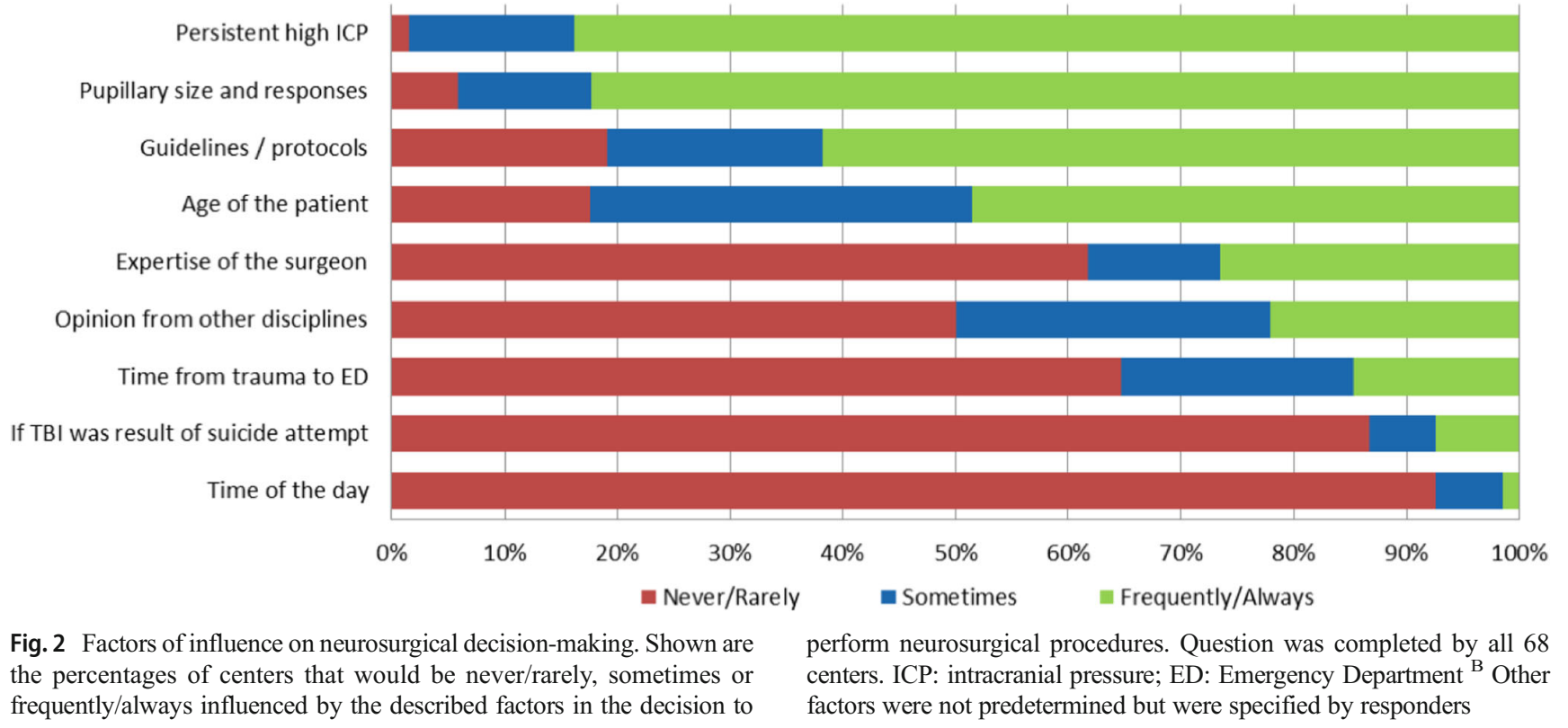


Table 2 Neurosurgical treatment policy of traumatic brain injury

Characteristic

Structural estimation of mass lesions on $\mathrm{CT}^{\mathrm{a}}$

Visual intuition (e.g., no actual measurement)

Width, diameter and/or amount of MLS of the mass lesion

Volume measurements with imaging software

Volume measurements with direct calculation

Other

ASDH operation determinants

Age considered important in surgery decision ${ }^{\mathrm{d}}$

Size (volume or thickness) threshold for surgery

Minimum volume or thickness:

$15 \mathrm{~mm}$

$10 \mathrm{~mm}$

$10 \mathrm{~mm}$ and/or $>5 \mathrm{~mm}$ MLS

$5 \mathrm{~mm}$

ASDH thickness $>$ width of cranium

Midline shift > thickness ASDH

DC indications

Routine

Intra-operative brain swelling

Sometimes as a second procedure in case of uncontrollable ICP

Never

$\mathrm{ICH} /$ contusion operation determinants

General policy

Pre-emptive (to prevent deterioration)

Delayed (after deterioration)

Variable (depends on surgeon)

Other

DC indications

Routine

Intra-operative brain swelling

Sometimes as a delayed procedure in case of uncontrollable ICP

Never

Raised ICP determinants

DC employed $>70 \%$ of refractory high ICP cases

Mostly early DC (within 6-12 h of refractory ICP)

Mostly late DC (as last resort to control ICP)

ICP threshold for DC

Raised ICP threshold for DC ( $\mathrm{mmHg})$ :

30

25

20

15

Not standardized

DC indications considered ${ }^{\mathrm{a}}$

Pre-emptive in raised ICP (not last resort)

Refractory raised ICP (last resort)

CT evidence of raised ICP

Intra-operative brain swelling

Routine with every ASDH or ICH evacuation
$N$ completed

No. $(\%)$ or mean (sd)

68

$11(16)$

17 (25)

$1(2)$

68

$26(42)$

27 (40)

$28^{\mathrm{b}}$

16 (24)

2 (3)

3 (4)

3 (4)

2 (3)

68

59 (86)

5 (7)

0 (0)

68

45 (66)

18 (27)

3 (4)

68

55 (81)

10 (15)

2 (3)

68

32 (46)

32 (47)

32 (47)

65 (96)

$64^{\mathrm{c}}$

11 (17)

1 (2)

1 (2)

7 (10)

68

64 (91)

45 (66)

2 (3) 
Table 2 (continued)

Characteristic

Policy towards extremity limb fractures ${ }^{\mathrm{e}}$

Damage control

Definitive care
$N$ completed

No. $(\%)$ or mean $(\mathrm{sd})$

MLS midline shift, BTF Brain Trauma Foundation, ICP intracranial pressure, hrs hours

${ }^{a}$ Multiple options possible

${ }^{\mathrm{b}}$ One responder did not report a threshold for surgery while answering a specific threshold $(10 \mathrm{~mm})$

${ }^{\mathrm{c}}$ One responder reported to employ a threshold for DC in raised ICP while not giving their specific threshold

${ }^{d}$ The question was whether the responder considers if the decision on surgery in acute SDH is influenced by age (based on a general consensus in their respective center)

${ }^{\mathrm{e}}$ Damage control is focused on the TBI. All extremity fractures are stabilized, but definitive treatment delayed. Definitive care: the extremity fractures are operated as soon as possible

\section{Contemporary neurosurgical care}

There are no recent comparable studies providing an overview of neurosurgical management on this scale. Two recent national surveys, in The United Kingdom and the Republic of Ireland and The Netherlands, have shown a comparable variability among neurosurgeons regarding the decision to evacuate an ASDH or to perform a primary DC [21,34].

When comparing our results to existing - much older - surveys, evacuation of a traumatic ICH seems to be less often considered than in the past $[11,30]$. Our results are concordant with older surveys in reporting variable use of DC for refractory raised ICP, despite the DECRA trial (the RECUEicp was not published

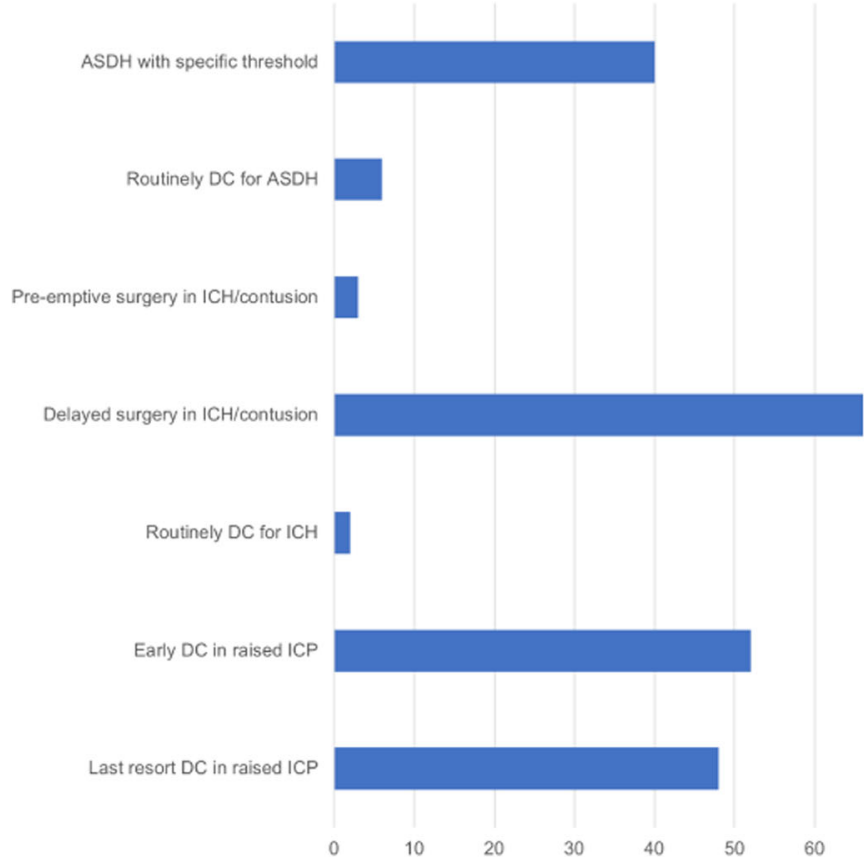

Fig. 3 Treatment indications for neurosurgical interventions. Shown are the proportions of centers that generally have these specific preferences with regard to operating or not in $\mathrm{ASDH}, \mathrm{ICH}$, and raised intracranial yet) $[12,19]$. Interestingly, although the mostly applied cutoff for DC in refractory is reported to be $25 \mathrm{mmHg}(60 \%)$, a lower value, $20 \mathrm{mmHg}$, and a higher value, $30 \mathrm{mmHg}$, are both reported to be used in almost $20 \%$ of centers.

More broadly, our results replicate past data that suggest poor guideline adherence and practice variability. Rayan et al. showed that in only $17 \%$ of a random sample of (brain) trauma patients care was delivered according to the BTF guidelines [31]. Of note, in the current study, surveys were sent to the centers between 2014 and 2015, so the more recent, updated BTF guidelines were not published yet, although the update was for medical management mainly (except DC in refractory IC) [6].

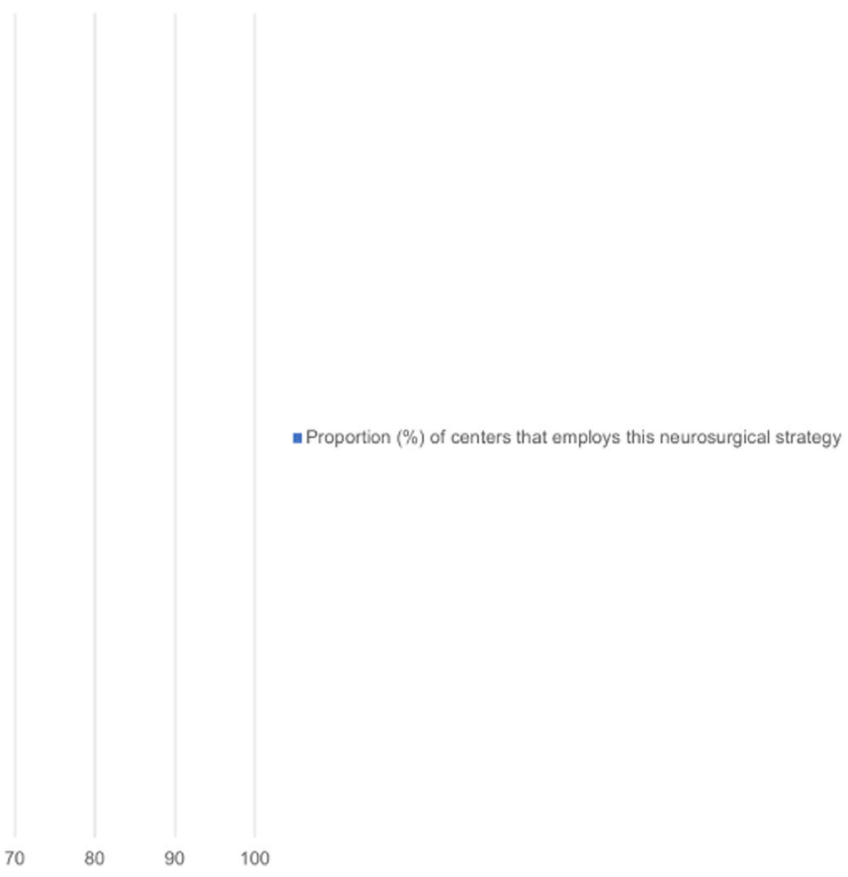

pressure, respectively. ASDH: acute subdural hematoma; DC: decompressive craniectomy; ICH: intracerebral hematoma; ICP: intracranial pressure 


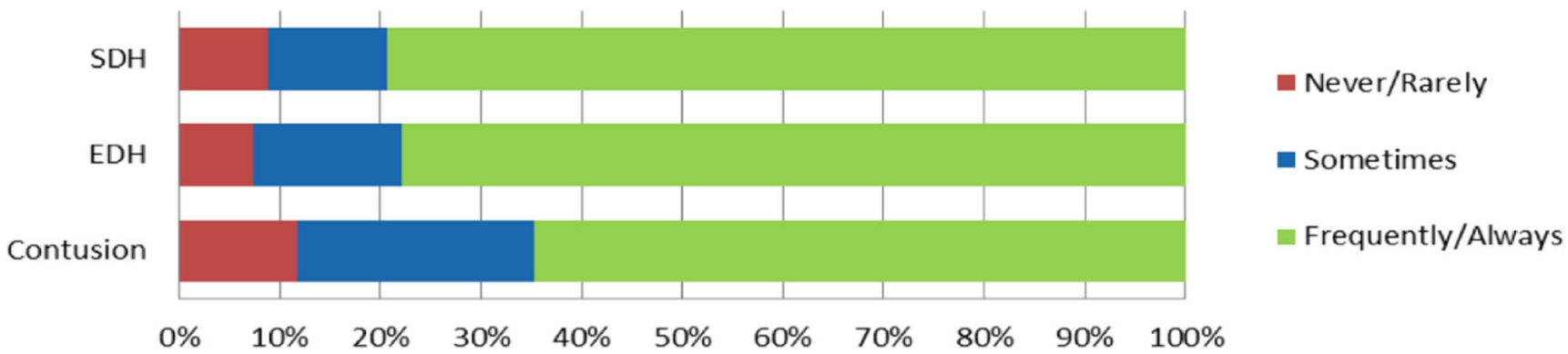

Fig. 4 BTF guideline adherence. Shown are the percentages of centers that reported to never/rarely, sometimes or frequently/always follow the Brain Trauma Foundation guidelines for the management of SDH, EDH, or contusions. Question was completed by 68 of the 68 centers. TBI: traumatic brain injury; SDH: subdural hematoma; EDH: epidural hematoma
Comparable questionnaires on other aspects of TBI care have recently been published for ER and ICU management that, without exception, show practice variation $[8,9,14,18]$. Practice variation has also been reported for other lifethreatening or emergency disorders including ruptured abdominal aneurysm [4] and the spontaneous intracerebral hemorrhage [16].

\section{Strengths and limitations}

A strength of the current study is the methodology that we used to investigate practice variation. First, detailed questions were posed to shed light on specific clinical decisions with regard to neurosurgical interventions. Subsequently, (objective) answers on amounts (volume load, mostly from in-hospital registries) were combined with qualitative information (estimations of general policies, using two approaches). When integrated with the high response rate and low amount of missing data in 68 centers, this overview provides a complete picture of reported neurosurgical care across Europe.

This study also had weaknesses. First, responses to the questionnaire may have been biased by the abstract nature of the questions posed, which neglected to provide a more concrete clinical context for judgments about reported practice.
Although the respondents were experienced neurosurgeons with a scientific background, the difficulty of weighing individual patient characteristics with potentially fatal consequences can never be fully captured by a theoretical survey. In particular, the rational decision-making can obviously be completely different due to the cognitive biases of neurosurgeons in the acute critical care period.

Second, there might be a concern as to how well the individual neurosurgeon respondent can represent the general center neurosurgical policy. Although we urged the respondent to report the general consensus on treatment at their center rather than individual management preferences (see Supplementary file 1), neurosurgical strategies may still be variable within centers between neurosurgeons; however, we did capture a qualitative assessment of this intra-center variability (Table 4). Third, we did not fully account for inherent regional variations such as evidence knowledge, caseload, and casemix due to referral patterns or admission policies, as a potential explanation for differences in neurosurgery policies. Variations in evidence knowledge for some questions, such as those on guidelines, are important. Moreover, while we did asses the center's caseload and casemix, the caseload and casemix of the (individual) respondent was not specifically asked. Fourth, the questions dealt with individual decisions in isolation, rather than the more complex real-life situation

Table 3 Within- and between-region variation in surgical management

\begin{tabular}{|c|c|c|c|c|c|c|c|c|}
\hline Decision & $\begin{array}{l}\text { Northern } \\
\text { Europe }\end{array}$ & $\begin{array}{l}\text { Western } \\
\text { Europe }\end{array}$ & $\begin{array}{l}\text { United } \\
\text { Kingdom }\end{array}$ & $\begin{array}{l}\text { Southern } \\
\text { Europe }\end{array}$ & $\begin{array}{l}\text { Eastern } \\
\text { Europe }\end{array}$ & $\begin{array}{l}\text { Baltic } \\
\text { States }\end{array}$ & Israel & $\begin{array}{l}\text { Nagelkerke } \mathrm{R}^{2} \\
\text { value }\end{array}$ \\
\hline \multicolumn{9}{|l|}{$A S D H$} \\
\hline - Size threshold for evacuation & 56 & 29 & 0 & 29 & 71 & 80 & 100 & 0.34 \\
\hline $\begin{array}{l}\text { - Routine or intraoperative DC } \\
\text { ICH/contusion }\end{array}$ & 89 & 92 & 100 & 100 & 86 & 80 & 100 & 0.17 \\
\hline $\begin{array}{l}\text { - Pre-emptive surgery Refractory } \\
\text { raised ICP }\end{array}$ & 0 & 0 & 0 & 7 & 0 & 20 & 0 & 0.35 \\
\hline$-\mathrm{DC}$ & 44 & 37 & 29 & 57 & 43 & 80 & 100 & 0.15 \\
\hline
\end{tabular}

$A S D H$ acute subdural hematoma, $I C H$ intracerebral hematoma, $D C$ decompressive craniectomy, $I C P$ intracranial pressure

Table presents the proportion $(\%)$ of respondent within each region that indicated that they used the described strategy as their general policy for patients with respectively ASDH, ICH, or refractory raised ICP. The Nagelkerke $\mathrm{R}^{2}$ value represents the variation in treatment that can be explained by the region 
Table 4 Neurosurgical decision making

\begin{tabular}{lll}
\hline Characteristic & $N$ completed & No $(\%)$ \\
\hline Structural variation ${ }^{\text {a }}$ ICP monitor insertion & 68 & \\
No & & $47(69)$ \\
Yes & & $21(31)$ \\
Structural variation ${ }^{\text {a }}$ mass lesion evacuation & 65 & \\
No & & $29(43)$ \\
Yes & $29(43)$ \\
Depending on lesion type & $7(10)$ \\
\hline
\end{tabular}

ED emergency department, GCS Glasgow Coma Scale

${ }^{\text {a }}$ Structural variation refers to a situation in which one or more of the clinicians are generally more likely to perform the (diagnostic) intervention than others

where several competing priorities need to be addressed. Fifth, the reports may have been biased (in varying extents) towards how centers would have been liked to be perceived, rather than a faithful report of actual clinical policy and practice. This issue will be addressed by a planned comparison of these Provider Profiling responses with actual treatment strategies employed in patient-level data from these centers in the CENTER-TBI Core study.

Finally, our study sample represents centers participating in TBI-research which are likely specialized neurotrauma centers with a tendency to have practice that is skewed towards up-todate knowledge. An example is the fact that almost half of all centers stated to have a neurosurgeon in house $24 \mathrm{~h}$ a day. When studying all centers in Europe providing care to TBI patients, variability might be even larger.

\section{Implications}

Our results should be interpreted in combination with the current evidence on the effectiveness of different surgical strategies. For the use of DC in refractory raised ICP due to diffuse swelling, two RCTs have provided useful guidance. The DECRA trial showed that early use of DC for modest rises in ICP was associated with worse outcomes [12]. More recently however, after the conduct of this survey, the RESCUEicp trial showed that, when used for refractory severe intracranial hypertension, DC can save lives, but results in an excess of severely disabled survivors [19]. It is clear that the intervention is not uniformly beneficial: while some functional improvements occur by 12 months, many survivors remain severely disabled. Rescue-ICP was not published yet at the conduct of this study. In our study, the majority of centers indicated that DC is often employed for both indications (preemptive and last resort).

With regard to focal lesions, a recent study suggested that in patients with an ASDH an aggressive approach towards evacuation is associated with better outcome compared to a conservative approach [35]. Similar trends were noted in the STITCH-trauma trial, which suggested better outcome with early surgical management of ICH [29]. In our study, a minority of centers considers an early strategy for ICH evacuation.

Lastly, DC in the primary evacuation of an ASDH seems to be associated with more favorable outcomes [22]. There is no class 1 evidence, although the research question is currently being challenged in an RCT (Rescue-ASDH; ISRCT87370545). In the current survey standard (in some cases preventive) DC in ASDH evacuation is rarely employed but mostly done in case of intraoperative swelling.

There may be several explanations for the practice variation that we observed. Although high practice variation rates can be a sign of poor implementation of evidence-based care, in this context it probably reflects the lack of strong evidence to underpin practice. In such a low evidence context, clinical decisions are not driven by careful consideration or penetration of the evidence, but by local customs and surgical training, handed down over the years from one surgeon to the other in a given center (or country). The professional cultural drivers that underpin such learned treatment preferences are resistant to change, and provide an important hurdle to the design and conduct of randomized studies for neurosurgical interventions in TBI [27].

Additionally, even where the results of RCTs are available, it is possible that many neurosurgeons do not think the RCT results applicable to their (individual) patients, or restrict their focus to short term clinical outcomes such as mortality and complication rates (instead of long-term clinical or patient reported outcomes). [13]

The results of the questionnaire point out burning clinical questions for neurosurgery in TBI. For ASDH and ICH, important questions include whether to operate or not, the timing of operative evacuation, and whether or not a primary DC should be undertaken. Future studies should address these questions. For DC, the variation should lead to studies exploring the lack of evidence penetration, in addition to studying effectiveness of DC in refractory raised ICP.

While RCTs may provide the security of randomization as a basis for examining answering these questions, RCTs have no successful history in TBI due to various reasons [24]. The CENTER-TBI Provider Profiling exercise has revealed large practice variation that can be related to variation in patient outcome [23]. Such a CER approach may be a pragmatic alternative to RCTs.

Therefore, different steps are required. Firstly, to specify, ideally a-priori, how and where treatment variation occurs. This was one of the goals of this provider profiling. Secondly, the CENTER-TBI Core Study will need to collect patient-level data from a large variety of centers, capturing the range of treatment variation and relate it to outcome. The main challenge is to disentangle the effect of specific surgical strategies in a center from other regional care variation that might 
affect outcome. To do so, we propose random-effect models in which the effect of "surgical strategy" on outcome is estimated with adjustment for other between-hospital differences in a random effect for hospital [10, 34, 35].

\section{Conclusions}

This survey study explored differences in neurosurgical strategies for TBI. Current neurosurgical care differs within Europe (and Israel), while the organization of trauma centers does not. This variation in practice likely reflects the lack of high-quality evidence for these important, potentially life-saving, emergency neurosurgical interventions. In addition, local professional culture may drive practice in ways that are not dependent on the availability or penetration of evidence. The resulting entrenched practice variation does not facilitate equipoise that makes RCTs easy to deliver. CER may provide a pragmatic approach to generate evidence on optimal neurosurgical strategies for TBI patients.

Funding/sponsors This study was funded by the European Union Seventh Framework Program (grant 602150) for Collaborative European NeuroTrauma Effectiveness Research in Traumatic Brain Injury (CENTER-TBI) and the Hersenstichting Nederland (Dutch Brain Foundation, grant PS2014-06) for The Dutch Neurotraumatology Quality Registry (Net-QuRe). There is no industry affiliation.

\section{Compliance with ethical standards}

Conflict of interest The authors declare that they have no conflicts of interest.

All procedures performed in studies involving human participants were in accordance with ethical standards of the institutional and national research committee and with the 1964 Helsinki declaration and its later amendments.

\section{Appendix}

The CENTER-TBI Investigators and Participants and their affiliations:

Ackerlund Cecilia ${ }^{1}$, Adams Hadie ${ }^{2}$, Agnoletti Vanni ${ }^{3}$, Allanson Judith ${ }^{4}$, Amrein Krisztina ${ }^{5}$, Andaluz Norberto ${ }^{6}$, Andelic $\mathrm{Nada}^{7}$, Andreassen Lasse ${ }^{8}$, Antun Azasevac ${ }^{9}$, Anke Audny ${ }^{10}$, Antoni Anna ${ }^{11}$, Ardon Hilko ${ }^{12}$, Audibert Gérard ${ }^{13}$, Auslands Kaspars ${ }^{14}$, Azouvi Philippe ${ }^{15}$, Azzolini Maria Luisa ${ }^{16}$, Baciu Camelia ${ }^{17}$, Badenes Rafael ${ }^{18}$, Bartels Ronald ${ }^{19}$, Barzó Pál ${ }^{20}$, Bauerfeind Ursula ${ }^{21}$, Beauvais Romuald ${ }^{22}$, Beer Ronny ${ }^{23}$, Belda Francisco Javier ${ }^{18}$, Bellander BoMichael ${ }^{24}$, Belli Antonio ${ }^{25}$, Bellier Rémy ${ }^{26}$, Benali Habib ${ }^{27}$, Benard Thierry ${ }^{26}$, Berardino Maurizio ${ }^{28}$, Beretta Luigi ${ }^{16}$, Beynon Christopher ${ }^{29}$, Bilotta Federico ${ }^{18}$, Binder Harald ${ }^{11}$, Biqiri Erta ${ }^{17}$, Blaabjerg Morten ${ }^{30}$, den Boogert Hugo ${ }^{19}$,
Bouzat Pierre ${ }^{31}$, Bragge Peter ${ }^{32}$, Brazinova Alexandra ${ }^{33}$, Brinck Vibeke ${ }^{34}$, Brooker Joanne ${ }^{35}$, Brorsson Camilla ${ }^{36}$, Buki Andras ${ }^{37}$, Bullinger Monika ${ }^{38}$, Calappi Emiliana ${ }^{39}$, Calvi Maria Rosa ${ }^{16}$, Cameron Peter ${ }^{40}$, Carbayo Lozano Guillermo $^{41}$, Carbonara Marco ${ }^{39}$, Carise Elsa ${ }^{26}$, Carpenter K. ${ }^{42}$, Castaño-León Ana M. ${ }^{43}$, Causin Francesco ${ }^{44}$, Chevallard Giorgio ${ }^{17}$, Chieregato Arturo ${ }^{17}$, Citerio Giuseppe ${ }^{45}$, 46 , Cnossen Maryse ${ }^{47}$, Coburn Mark ${ }^{48}$, Coles Jonathan $^{49}$, Coles-Kemp Lizzie ${ }^{50}$, Collett Johnny ${ }^{50}$, Cooper Jamie D. ${ }^{51}$, Correia Marta ${ }^{52}$, Covic Amra ${ }^{53}$, Curry Nicola ${ }^{54}$, Czeiter Endre ${ }^{55}$, Czosnyka Marek ${ }^{56}$, Dahyot-Fizelier Claire ${ }^{26}$, Damas François ${ }^{57}$, Damas Pierre ${ }^{58}$, Dawes Helen ${ }^{59}$, De Keyser Véronique ${ }^{60}$, Della Corte Francesco ${ }^{61}$, Depreitere Bart ${ }^{62}$, de Ruiter Godard C.W. ${ }^{63}$, Dilvesi Dula ${ }^{9}$, Ding Shenghao ${ }^{64}$, Dippel Diederik ${ }^{65}$, Dixit Abhishek ${ }^{66}$, Donoghue Emma ${ }^{40}$, Dreier Jens ${ }^{67}$, Dulière Guy-Loup ${ }^{57}$, Eapen George ${ }^{68}$, Engemann Heiko ${ }^{53}$, Ercole Ari ${ }^{66}$, Esser Patrick ${ }^{59}$, Ezer Erzsébet ${ }^{69}$, Fabricius Martin ${ }^{70}$, Feigin Valery L. ${ }^{71}$, Feng Junfeng ${ }^{64}$, Foks Kelly ${ }^{65}$, Fossi Francesca ${ }^{17}$, Francony Gilles ${ }^{31}$, Freo Ulderico ${ }^{72}$, Frisvold Shirin ${ }^{73}$, Furmanov Alex ${ }^{74}$, Gagliardo Pablo ${ }^{75}$, Galanaud Damien ${ }^{27}$, Gantner Dashiell ${ }^{40}$, Gao Guoyi ${ }^{76}$, Geleijns Karin ${ }^{42}$, George Pradeep ${ }^{1}$, Ghuysen Alexandre ${ }^{77}$, Giga Lelde ${ }^{78}$, Giraud Benoit $^{26}$, Glocker Ben ${ }^{79}$, Golubovic Jagos ${ }^{9}$, Gomez Pedro A. ${ }^{43}$, Grossi Francesca ${ }^{61}$, Gruen Russell L. ${ }^{80}$, Gupta Deepak ${ }^{81}$, Haagsma Juanita A. ${ }^{47}$, Haitsma Iain ${ }^{82}$, Hartings Jed A. ${ }^{83}$, Helbok Raimund ${ }^{23}$, Helseth Eirik ${ }^{84}$, Hertle Daniel ${ }^{30}$, Hoedemaekers Astrid ${ }^{85}$, Hoefer Stefan ${ }^{53}$, Horton Lindsay ${ }^{86}$, Huijben Jilske ${ }^{47}$, Hutchinson Peter J. ${ }^{2}$, Håberg Asta Kristine $^{87}$, Jacobs Bram ${ }^{88}$, Jankowski Stefan ${ }^{68}$, Jarrett Mike $^{34}$, Jelaca Bojan ${ }^{9}$, Jiang Ji-yao ${ }^{76}$, Jones Kelly ${ }^{89}$, Kamnitsas Konstantinos ${ }^{79}$, Karan Mladen ${ }^{6}$, Katila Ari ${ }^{90}$, Kaukonen Maija ${ }^{91}$, Kerforne Thomas ${ }^{26}$, Kivisaari Riku ${ }^{91}$, Kolias Angelos G. ${ }^{2}$, Kolumbán Bálint ${ }^{92}$, Kompanje Erwin ${ }^{93}$, Kolundzija Ksenija ${ }^{94}$, Kondziella Daniel ${ }^{70}$, Koskinen Lars-Owe ${ }^{36}$, Kovács Noémi ${ }^{92}$, Lagares Alfonso ${ }^{43}$, Lanyon Linda ${ }^{1}$, Laureys Steven ${ }^{95}$, Lecky Fiona ${ }^{96}$, Ledig Christian ${ }^{79}$, Lefering Rolf ${ }^{97}$, Legrand Valerie ${ }^{98}$, Lei Jin ${ }^{64}$, Levi Leon ${ }^{99}$, Lightfoot Roger ${ }^{100}$, Lingsma Hester ${ }^{47}$, Loeckx Dirk ${ }^{101}$, Lozano Angels ${ }^{18}$, Maas Andrew I.R. ${ }^{60}$, MacDonald Stephen ${ }^{102}$, Maegele Marc ${ }^{103}$, Majdan Marek ${ }^{33}$, Major Sebastian ${ }^{104}$, Manara Alex ${ }^{105}$, Manley Geoffrey ${ }^{106}$, Martin Didier ${ }^{107}$, Martin Leon Francisco ${ }^{101}$, Martino Costanza ${ }^{3}$, Maruenda Armando ${ }^{18}$, Maréchal Hugues ${ }^{57}$, Masala Alessandro ${ }^{3}$, Mattern Julia ${ }^{29}$, McFadyen Charles ${ }^{66}$, McMahon Catherine ${ }^{108}$, Melegh Béla ${ }^{109}$, Menon David ${ }^{66}$, Menovsky Tomas ${ }^{60}$, Morganti-Kossmann Cristina ${ }^{110}$, Mulazzi Davide ${ }^{39}$, Muraleedharan Visakh ${ }^{1}$, Murray Lynnette ${ }^{40}$, Mühlan Holger ${ }^{111}$, Nair Nandesh ${ }^{60}$, Negru Ancuta ${ }^{112}$, Nelson David ${ }^{1}$, Newcombe Virginia ${ }^{66}$, Nieboer Daan ${ }^{47}$, Noirhomme Quentin $^{95}$, Nyirádi József ${ }^{5}$, Oddo Mauro ${ }^{113}$, Oldenbeuving Annemarie ${ }^{114}$, Oresic Matej ${ }^{115}$, Ortolano Fabrizio ${ }^{39}$, Palotie Aarno 116, 117, 118 , Parizel Paul M. ${ }^{119}$, Patruno Adriana ${ }^{120}$, 
Payen Jean-François ${ }^{31}$, Perera Natascha ${ }^{22}$, Perlbarg Vincent 27 , Persona Paolo ${ }^{121}$, Peul Wilco ${ }^{63}$, Piippo-Karjalainen Anna ${ }^{91}$, Pili Floury Sébastien ${ }^{122}$, Pirinen Matti ${ }^{116}$, Ples Horia ${ }^{112}$, Poca Maria Antonia ${ }^{123}$, Polinder Suzanne ${ }^{47}$, Pomposo Inigo ${ }^{41}$, Posti Jussi ${ }^{90}$, Puybasset Louis ${ }^{124}$, Radoi Andreea ${ }^{123}$, Ragauskas Arminas ${ }^{125}$, Raj Rahul ${ }^{91}$, Rambadagalla Malinka ${ }^{126}$, Real Ruben ${ }^{53}$, Rehorčíková Veronika ${ }^{33}$, Rhodes Jonathan ${ }^{127}$, Ripatti Samuli ${ }^{116}$, Rocka Saulius ${ }^{125}$, Roe Cecilie ${ }^{128}$, Roise Olav ${ }^{129}$, Roks Gerwin ${ }^{130}$, Rosand Jonathan ${ }^{131}$, Rosenfeld Jeffrey ${ }^{110}$, Rosenlund Christina ${ }^{132}$, Rosenthal Guy ${ }^{74}$, Rossaint Rolf ${ }^{48}$, Rossi Sandra ${ }^{121}$, Rueckert Daniel ${ }^{79}$, Rusnák Martin ${ }^{133}$, Sacchi Marco 17, Sahakian Barbara ${ }^{66}$, Sahuquillo Juan ${ }^{123}$, Sakowitz Oliver 134, 135, Sala Francesca ${ }^{120}$, Sanchez-Porras Renan ${ }^{134}$, Sandor Janos ${ }^{136}$, Santos Edgar ${ }^{29}$, Sasu Luminita ${ }^{61}$, Savo Davide $^{120}$, Schäffer Nadine ${ }^{103}$, Schipper Inger ${ }^{137}$, Schlößer Barbara ${ }^{21}$, Schmidt Silke ${ }^{111}$, Schoechl Herbert ${ }^{138}$, Schoonman Guus ${ }^{130}$, Schou Rico Frederik ${ }^{139}$, Schwendenwein Elisabeth ${ }^{11}$, Schöll Michael ${ }^{29}$, Sir Özcan ${ }^{140}$, Skandsen Toril ${ }^{141}$, Smakman Lidwien ${ }^{63}$, Smeets Dirk ${ }^{101}$, Smielewski Peter ${ }^{56}$, Sorinola Abayomi ${ }^{142}$, Stamatakis Emmanuel ${ }^{66}$, Stanworth Simon ${ }^{54}$, Steinbüchel Nicole ${ }^{143}$, Stevanovic Ana ${ }^{48}$, Stevens Robert ${ }^{144}$, Stewart William ${ }^{145}$, Steyerberg Ewout W. ${ }^{47,146}$, Stocchetti Nino ${ }^{147}$, Sundström Nina ${ }^{36}$, Synnot Anneliese ${ }^{34,148}$, Taccone Fabio Silvio ${ }^{18}$, Takala Riikka ${ }^{90}$, Tamás Viktória ${ }^{142}$, Tanskanen Päivi ${ }^{91}$, Taylor Mark Steven ${ }^{33}$, Te Ao Braden ${ }^{71}$, Tenovuo Olli ${ }^{90}$, Telgmann Ralph ${ }^{53}$, Teodorani Guido ${ }^{149}$, Theadom Alice ${ }^{71}$, Thomas Matt ${ }^{105}$, Tibboel Dick ${ }^{42}$, Tolias Christos ${ }^{150}$, Tshibanda Jean-Flory Luaba ${ }^{151}$, Trapani Tony ${ }^{40}$, Tudora Cristina Maria ${ }^{112}$, Vajkoczy Peter ${ }^{67}$, Vallance Shirley ${ }^{43}$, Valeinis Egils ${ }^{78}$, Van der Steen Gregory ${ }^{60}$, van der Jagt Mathieu ${ }^{152}$, van der Naalt Joukje ${ }^{88}$, van Dijck Jeroen T.J.M. ${ }^{63}$, van Essen Thomas A. ${ }^{63}$, Van Hecke Wim ${ }^{101}$, van Heugten Caroline ${ }^{59}$, Van Praag Dominique ${ }^{60}$, Vande Vyvere Thijs ${ }^{101}$, Van Waesberghe Julia ${ }^{48}$, Vanhaudenhuyse Audrey 27, 95, Vargiolu Alessia ${ }^{120}$, Vega Emmanuel ${ }^{153}$, Velt Kimberley ${ }^{47}$, Verheyden Jan ${ }^{101}$, Vespa Paul M. ${ }^{154}$, Vik Anne ${ }^{155}$, Vilcinis Rimantas ${ }^{156}$, Vizzino Giacinta ${ }^{17}$, Vleggeert-Lankamp Carmen ${ }^{63}$, Volovici Victor ${ }^{82}$, Voormolen Daphne ${ }^{47}$, Vulekovic Peter ${ }^{9}$, Vámos Zoltán ${ }^{69}$, Wade Derick $^{59}$, Wang Kevin K.W. ${ }^{157}$, Wang Lei ${ }^{64}$, Wessels Lars ${ }^{158}$, Wildschut Eno ${ }^{42}$, Williams Guy ${ }^{66}$, Wilson Lindsay ${ }^{86}$, Winkler Maren K.L. ${ }^{104}$, Wolf Stefan ${ }^{158}$, Ylén Peter ${ }^{159}$, Younsi Alexander ${ }^{29}$, Zaaroor Menashe ${ }^{99}$, Zhihui Yang ${ }^{160}$, Ziverte Agate ${ }^{78}$, Zumbo Fabrizio ${ }^{3}$.

1 Karolinska Institutet, INCF International Neuroinformatics Coordinating Facility, Stockholm, Sweden.

2 Division of Neurosurgery, Department of Clinical Neurosciences, Addenbrooke's Hospital \& University of Cambridge, Cambridge, UK.

${ }^{3}$ Department of Anesthesia \& Intensive Care, M. Bufalini Hospital, Cesena, Italy.
${ }^{4}$ Department of Clinical Neurosciences, Addenbrooke's Hospital \& University of Cambridge, Cambridge, UK.

5 János Szentágothai Research Centre, University of Pécs, Pécs, Hungary.

${ }^{6}$ University of Cincinnati, Cincinnati, Ohio, United States.

7 Division of Surgery and Clinical Neuroscience, Department of Physical Medicine and Rehabilitation, Oslo University Hospital and University of Oslo, Oslo, Norway.

${ }^{8}$ Department of Neurosurgery, University Hospital Northern Norway, Tromso, Norway.

9 Department of Neurosurgery, Clinical centre of Vojvodina, Faculty of Medicine, University of Novi Sad, Novi Sad, Serbia.

${ }^{10}$ Department of Physical Medicine and Rehabilitation, University hospital Northern Norway.

11 Trauma Surgery, Medical University Vienna, Vienna, Austria.

${ }^{12}$ Department of Neurosurgery, Elisabeth-Tweesteden Ziekenhuis, Tilburg, the Netherlands.

13 Department of Anesthesiology \& Intensive Care, University Hospital Nancy, Nancy, France.

${ }^{14}$ Riga Eastern Clinical University Hospital, Riga, Latvia.

15 Raymond Poincare hospital, Assistance Publique Hopitaux de Paris, Paris, France.

16 Department of Anesthesiology \& Intensive Care, S

Raffaele University Hospital, Milan, Italy.

${ }^{17}$ NeuroIntensive Care, Niguarda Hospital.

18 Department Anesthesiology and Surgical-Trauma Intensive Care, Hospital Clinic Universitari de Valencia, Spain.

${ }^{19}$ Department of Neurosurgery, Radboud University Medical Center.

${ }^{20}$ Department of Neurosurgery, University of Szeged, Szeged, Hungary.

${ }^{21}$ Institute for Transfusion Medicine (ITM), Witten/ Herdecke University, Cologne, Germany.

${ }^{22}$ International Projects Management, ARTTIC, Munchen, Germany.

${ }^{23}$ Department of Neurology, Neurological Intensive Care Unit, Medical University of Innsbruck, Innsbruck, Austria.

${ }^{24}$ Department of Neurosurgery \& Anesthesia \& intensive care medicine, Karolinska University Hospital, Stockholm, Sweden.

25 NIHR Surgical Reconstruction and Microbiology Research Centre, Birmingham, UK.

${ }^{26}$ Intensive care Unit, CHU Poitiers, Poitiers, France.

27 Anesthesie-Réanimation, Assistance Publique Hopitaux de Paris, Paris, France.

28 Department of Anesthesia \& ICU, AOU Città della Salute e della Scienza di Torino - Orthopedic and Trauma Center, Torino, Italy.

29 Department of Neurosurgery, University Hospital Heidelberg, Heidelberg, Germany. 
${ }^{30}$ Department of Neurology, Odense University Hospital, Odense, Denmark.

31 Department of Anesthesiology \& Intensive Care, University Hospital of Grenoble, Grenoble, France.

32 BehaviourWorks Australia, Monash Sustainability Institute, Monash University, Victoria, Australia.

${ }^{33}$ Department of Public Health, Faculty of Health Sciences and Social Work, Trnava University, Trnava, Slovakia.

34 Quesgen Systems Inc., Burlingame, California, USA.

35 Australian \& New Zealand Intensive Care Research Centre, Department of Epidemiology and Preventive Medicine, School of Public Health and Preventive Medicine, Monash University, Melbourne, Australia.

${ }^{36}$ Department of Neurosurgery, Umea University Hospital, Umea, Sweden.

37 Department of Neurosurgery, University of Pecs and MTA-PTE Clinical Neuroscience MR Research Group and Janos Szentagothai Research Centre, University of Pecs, Hungarian Brain Research Program, Pecs, Hungary.

38 Department of Medical Psychology, Universitätsklinikum Hamburg-Eppendorf, Hamburg, Germany.

39 Neuro ICU, Fondazione IRCCS Cà Granda Ospedale Maggiore Policlinico, Milan, Italy.

40 ANZIC Research Centre, Monash University, Department of Epidemiology and Preventive Medicine, Melbourne, Vitoria, Australia.

41 Department of Neurosurgery, Hospital of Cruces, Bilbao, Spain.

42 Intensive Care and Department of Pediatric Surgery,

Erasmus Medical Center, Sophia Children's Hospital, Rotterdam, The Netherlands.

${ }^{43}$ Department of Neurosurgery, Hospital Universitario 12 de Octubre, Madrid, Spain.

44 Department of Neuroscience, Azienda Ospedaliera

Università di Padova, Padova, Italy.

${ }^{45}$ NeuroIntensive Care, ASST di Monza, Monza, Italy.

46 School of Medicine and Surgery, Università Milano Bicocca, Milano, Italy.

${ }^{47}$ Department of Public Health, Erasmus Medical CenterUniversity Medical Center, Rotterdam, The Netherlands.

48 Department of Anaesthesiology, University Hospital of Aachen, Aachen, Germany.

49 Department of Anesthesia \& Neurointensive Care, Cambridge University Hospital NHS Foundation Trust, Cambridge, UK.

${ }^{50}$ Movement Science Group, Oxford Institute of Nursing, Midwifery and Allied Health Research, Oxford Brookes University, Oxford, UK.

${ }^{51}$ School of Public Health \& PM, Monash University and The Alfred Hospital, Melbourne, Victoria, Australia.

52 Radiology/MRI department, MRC Cognition and Brain Sciences Unit, Cambridge, UK.
53 Institute of Medical Psychology and Medical Sociology, Universitätsmedizin Göttingen, Göttingen, Germany.

${ }^{54}$ Oxford University Hospitals NHS Trust, Oxford, UK.

55 Department of Neurosurgery, University of Pecs and MTA-PTE Clinical Neuroscience MR Research Group and Janos Szentagothai Research Centre, University of Pecs, Hungarian Brain Research Program (Grant No. KTIA 13 NAP-A-II/8), Pecs, Hungary.

56 Brain Physics Lab, Division of Neurosurgery, Dept of Clinical Neurosciences, University of Cambridge, Addenbrooke's Hospital, Cambridge, UK.

${ }^{57}$ Intensive Care Unit, CHR Citadelle, Liège, Belgium.

58 Intensive Care Unit, CHU, Liège, Belgium.

59 Movement Science Group, Faculty of Health and Life Sciences, Oxford Brookes University, Oxford, UK.

${ }^{60}$ Department of Neurosurgery, Antwerp University Hospital and University of Antwerp, Edegem, Belgium.

61 Department of Anesthesia \& Intensive Care, Maggiore

Della Carità Hospital, Novara, Italy.

${ }^{62}$ Department of Neurosurgery, University Hospitals

Leuven, Leuven, Belgium.

63 Dept. of Neurosurgery, Leiden University Medical Center, Leiden, The Netherlands and Dept. of Neurosurgery, Medical Center Haaglanden, The Hague, The Netherlands.

${ }^{64}$ Department of Neurosurgery, Renji Hospital, Shanghai

Jiaotong University School of Medicine, Shanghai, China.

${ }^{65}$ Department of Neurology, Erasmus MC, Rotterdam, the

Netherlands.

66 Division of Anaesthesia, University of Cambridge,

Addenbrooke's Hospital, Cambridge, UK.

${ }^{67}$ Neurologie, Neurochirurgie und Psychiatrie, Charité -

Universitätsmedizin Berlin, Berlin, Germany.

${ }^{68}$ Neurointensive Care, Sheffield Teaching Hospitals NHS Foundation Trust, Sheffield, UK.

${ }^{69}$ Department of Anaesthesiology and Intensive Therapy, University of Pécs, Pécs, Hungary.

${ }^{70}$ Departments of Neurology, Clinical Neurophysiology and Neuroanesthesiology, Region Hovedstaden Rigshospitalet, Copenhagen, Denmark.

${ }^{71}$ National Institute for Stroke and Applied Neurosciences, Faculty of Health and Environmental Studies, Auckland University of Technology, Auckland, New Zealand.

72 Department of Medicine, Azienda Ospedaliera Università di Padova, Padova, Italy.

73 Department of Anesthesiology and Intensive care, University Hospital Northern Norway, Tromso, Norway.

74 Department of Neurosurgery, Hadassah-hebrew University Medical center, Jerusalem, Israel.

${ }^{75}$ Fundación Instituto Valenciano de Neurorrehabilitación (FIVAN), Valencia, Spain.

${ }^{76}$ Department of Neurosurgery, Shanghai Renji hospital, Shanghai Jiaotong University/school of medicine, Shanghai, China. 
${ }^{77}$ Emergency Department, CHU, Liège, Belgium.

${ }^{78}$ Pauls Stradins Clinical University Hospital, Riga, Latvia.

${ }^{79}$ Department of Computing, Imperial College London, London, UK.

${ }^{80}$ Lee Kong Chian School of Medicine, Nanyang Technological University, Singapore; and Monash University, Australia.

${ }^{81}$ Department of Neurosurgery, Neurosciences Centre \& JPN Apex trauma centre, All India Institute of Medical Sciences, New Delhi-110,029, India.

${ }^{82}$ Department of Neurosurgery, Erasmus MC, Rotterdam, the Netherlands.

${ }^{83}$ Department of Neurosurgery, University of Cincinnati, Cincinnati, Ohio, USA.

${ }^{84}$ Department of Neurosurgery, Oslo University Hospital, Oslo, Norway.

${ }^{85}$ Department of Intensive Care Medicine, Radboud University Medical Center.

${ }^{86}$ Division of Psychology, University of Stirling, Stirling, UK.

${ }^{87}$ Department of Medical Imaging, St. Olavs Hospital and Department of Neuroscience, Norwegian University of Science and Technology, Trondheim, Norway.

${ }^{88}$ Department of Neurology, University Medical Center Groningen, Groningen, Netherlands.

${ }^{89}$ National Institute for Stroke \& Applied Neurosciences of the AUT University, Auckland, New Zealand.

${ }^{90}$ Rehabilitation and Brain Trauma, Turku University Central Hospital and University of Turku, Turku, Finland.

${ }^{91}$ Helsinki University Central Hospital.

${ }^{92}$ Hungarian Brain Research Program - Grant No. KTIA 13 NAP-A-II/8, University of Pécs, Pécs, Hungary.

${ }^{93}$ Department of Intensive Care and Department of Ethics and Philosophy of Medicine, Erasmus Medical Center, Rotterdam, The Netherlands.

${ }^{94}$ Department of Psichiatry, Clinical centre of Vojvodina, Faculty of Medicine, University of Novi Sad, Novi Sad, Serbia.

${ }^{95}$ Cyclotron Research Center, University of Liège, Liège, Belgium.

${ }^{96}$ Emergency Medicine Research in Sheffield, Health Services Research Section, School of Health and Related Research (ScHARR), University of Sheffield, Sheffield, UK.

${ }^{97}$ Institute of Research in Operative Medicine (IFOM), Witten/Herdecke University, Cologne, Germany.

${ }^{98}$ VP Global Project Management CNS, ICON, Paris, France.

${ }^{99}$ Department of Neurosurgery, Rambam Medical Center, Haifa, Israel.

${ }^{100}$ Department of Anesthesiology \& Intensive Care, University Hospitals Southhampton NHS Trust, Southhampton, UK.
101 icoMetrix NV, Leuven, Belgium.

102 Cambridge University Hospitals, Cambridge, UK.

103 Cologne-Merheim Medical Center (CMMC),

Department of Traumatology, Orthopedic Surgery and Sportmedicine, Witten/Herdecke University, Cologne, Germany.

104 Centrum für Schlaganfallforschung, Charité Universitätsmedizin Berlin, Berlin, Germany.

${ }^{105}$ Intensive Care Unit, Southmead Hospital, Bristol,

Bristol, UK.

${ }^{106}$ Department of Neurological Surgery, University of

California, San Francisco, California, USA.

${ }^{107}$ Department of Neurosurgery, CHU, Liège, Belgium.

${ }^{108}$ Department of Neurosurgery, The Walton centre NHS

Foundation Trust, Liverpool, UK.

${ }^{109}$ Department of Medical Genetics, University of Pécs,

Pécs, Hungary.

${ }^{110}$ National Trauma Research Institute, The Alfred

Hospital, Monash University, Melbourne, Victoria, Australia.

111 Department Health and Prevention, University

Greifswald, Greifswald, Germany.

${ }^{112}$ Department of Neurosurgery, Emergency County

Hospital Timisoara, Timisoara, Romania.

${ }^{113}$ Centre Hospitalier Universitaire Vaudois.

${ }^{114}$ Department of Intensive Care, Elisabeth-Tweesteden

Ziekenhuis, Tilburg, the Netherlands.

${ }^{115}$ Department of Systems Medicine, Steno Diabetes

Center, Gentofte, Denmark.

${ }^{116}$ Institute for Molecular Medicine Finland, University of

Helsinki, Helsinki, Finland.

117 Analytic and Translational Genetics Unit, Department

of Medicine; Psychiatric \& Neurodevelopmental Genetics

Unit, Department of Psychiatry; Department of Neurology,

Massachusetts General Hospital, Boston, MA, USA.

118 Program in Medical and Population Genetics; The

Stanley Center for Psychiatric Research, The Broad Institute of MIT and Harvard, Cambridge, MA, USA.

${ }^{119}$ Department of Radiology, Antwerp University Hospital and University of Antwerp, Edegem, Belgium.

${ }^{120}$ NeuroIntenisve Care Unit, Department of Anesthesia \&

Intensive Care, ASST di Monza, Monza, Italy.

${ }^{121}$ Department of Anesthesia \& Intensive Care, Azienda Ospedaliera Università di Padova, Padova, Italy.

${ }^{122}$ Intensive Care Unit, CHRU de Besançon, Besançon, France.

${ }^{123}$ Department of Neurosurgery, Vall d'Hebron University Hospital, Barcelona, Spain.

${ }^{124}$ Department of Anesthesiology and Critical Care, Pitié Salpêtrière Teaching Hospital, Assistance Publique, Hôpitaux de Paris and University Pierre et Marie Curie, Paris, France.

${ }^{125}$ Department of Neurosurgery, Kaunas University of technology and Vilnius University, Vilnius, Lithuania.

${ }^{126}$ Rezekne Hospital, Latvia. 
127 Department of Anesthesia, Critical Care \& Pain Medicine NHS Lothian \& University of Edinburg, Edinburgh, UK.

128 Department of Physical Medicine and Rehabilitation, Oslo University Hospital/University of Oslo, Oslo, Norway.

129 Division of Surgery and Clinical Neuroscience, Oslo University Hospital, Oslo, Norway.

130 Department of Neurology, Elisabeth-TweeSteden Ziekenhuis, Tilburg, the Netherlands.

131 Broad Institute, Cambridge MA Harvard Medical School, Boston MA, Massachusetts General Hospital, Boston MA, USA.

132 Department of Neurosurgery, Odense University Hospital, Odense, Denmark.

133 International Neurotrauma Research Organization, Vienna, Austria.

134 Klinik für Neurochirurgie, Klinikum Ludwigsburg, Ludwigsburg, Germany.

135 University Hospital Heidelberg, Heidelberg, Germany.

136 Division of Biostatistics and Epidemiology,

Department of Preventive Medicine, University of

Debrecen, Debrecen, Hungary.

137 Department of Traumasurgery, Leiden University

Medical Center, Leiden, The Netherlands.

138 Department of Anaesthesiology and Intensive Care,

AUVA Trauma Hospital, Salzburg, Austria.

139 Department of Neuroanesthesia and Neurointensive

Care, Odense University Hospital, Odense, Denmark.

140 Department of Emergency Care Medicine, Radboud

University Medical Center.

141 Department of Physical Medicine and Rehabilitation,

St. Olavs Hospital and Department of Neuroscience, Norwegian University of Science and Technology, Trondheim, Norway.

142 Department of Neurosurgery, University of Pécs, Pécs, Hungary.

${ }^{143}$ Universitätsmedizin Göttingen, Göttingen, Germany.

${ }^{144}$ Division of Neuroscience Critical Care, John Hopkins

University School of Medicine, Baltimore, USA.

145 Department of Neuropathology, Queen Elizabeth University Hospital and University of Glasgow, Glasgow, UK.

146 Dept. of Department of Biomedical Data Sciences, Leiden University Medical Center, Leiden.

147 Department of Pathophysiology and Transplantation, Milan University, and Neuroscience ICU, Fondazione IRCCS Cà Granda Ospedale Maggiore Policlinico, Milano, Italy.

148 Cochrane Consumers and Communication Review Group, Centre for Health Communication and Participation, School of Psychology and Public Health, La Trobe University, Melbourne, Australia.
149 Department of Reahabilitation, M. Bufalini Hospital, Cesena, Italy.

150 Department of Neurosurgery, Kings college London, London, UK.

${ }^{151}$ Radiology/MRI Department, CHU, Liège, Belgium.

152 Department of Intensive Care, Erasmus MC, Rotterdam, the Netherlands.

153 Department of Anesthesiology-Intensive Care, Lille

University Hospital, Lille, France.

${ }^{154}$ Director of Neurocritical Care, University of California,

Los Angeles, USA.

155 Department of Neurosurgery, St. Olavs Hospital and

Department of Neuroscience, Norwegian University of Science and Technology, Trondheim, Norway.

156 Department of Neurosurgery, Kaunas University of

Health Sciences, Kaunas, Lithuania.

157 Department of Psychiatry, University of Florida, Gainesville, Florida, USA.

${ }^{158}$ Interdisciplinary Neuro Intensive Care Unit, Charité -

Universitätsmedizin Berlin, Berlin, Germany.

${ }^{159}$ VTT Technical Research Centre, Tampere, Finland.

${ }^{160}$ University of Florida, Gainesville, Florida, USA

Open Access This article is distributed under the terms of the Creative Commons Attribution 4.0 International License (http:// creativecommons.org/licenses/by/4.0/), which permits unrestricted use, distribution, and reproduction in any medium, provided you give appropriate credit to the original author(s) and the source, provide a link to the Creative Commons license, and indicate if changes were made.

\section{References}

1. Albuquerque FC (2013) Intracranial pressure monitoring after blunt head injuries: conflicting opinions. World Neurosurg 79(5-6):598

2. Bosco JLF, Silliman RA, Thwin SS, Geiger AM, Buist DSM, Prout MN, Yood MU, Haque R, Wei F, Lash TL (2010) A most stubborn bias: no adjustment method fully resolves confounding by indication in observational studies. J Clin Epidemiol 63(1):64-74

3. Bragge P, Synnot A, Maas AI, Menon DK, Cooper DJ, Rosenfeld JV, Gruen RL (2016) A state-of-the-science overview of randomized controlled trials evaluating acute management of moderate-tosevere traumatic brain injury. J Neurotrauma 33(16):1461-1478

4. Brattheim BJ, Eikemo TA, Altreuther M, Landmark AD, Faxvaag A (2012) Regional disparities in incidence, handling and outcomes of patients with symptomatic and ruptured abdominal aortic aneurysms in Norway. Eur J Vasc Endovasc Surg 44(3):267-272

5. Bullock MR, Chesnut R, Ghajar J, Gordon D, Hartl R, Newell DW, Servadei F, Walters BC, Wilberger JE (2006) Introduction. Neurosurgery 58(Supplement):S2-1-S2-3

6. Carney N, Totten AM, O Reilly C, et al (2016) Guidelines for the management of severe traumatic brain injury, Fourth Edition. Neurosurgery 1

7. Chesnut RM, Temkin N, Carney N et al (2012) A trial of intracranial-pressure monitoring in traumatic brain injury. N Engl J Med 367(26):2471-2481 
8. Cnossen MC, Huijben JA, van der Jagt M, et al (2017) Variation in monitoring and treatment policies for intracranial hypertension in traumatic brain injury: a survey in 66 neurotrauma centers participating in the CENTER-TBI study. Crit Care 21(1):233

9. Cnossen MC, Polinder S, Lingsma HF, Maas AIR, Menon D, Steyerberg EW, CENTER-TBI Investigators and Participants (2016) Variation in structure and process of care in traumatic brain injury: provider profiles of European Neurotrauma centers participating in the CENTER-TBI study. PLoS One 11(8):e0161367

10. Cnossen MC, van Essen TA, Ceyisakar IE et al (2018) Adjusting for confounding by indication in observational studies: a case study in traumatic brain injury. Clinical Epidemiology 10:841-852

11. Compagnone C, Murray GD, Teasdale GM, Maas AIR, Esposito D, Princi P, D Avella D, Servadei F (2005) The management of patients with Intradural post-traumatic mass lesions: a multicenter survey of current approaches to surgical management in 729 patients coordinated by the European Brain Injury Consortium. Neurosurgery:1183-1192

12. Cooper DJ, Nichol A, Hodgson C (2016) Craniectomy for traumatic intracranial hypertension. N Engl J Med 375(24):2402

13. Ergina PL, Cook JA, Blazeby JM, Boutron I, Clavien P-A, Reeves BC, Seiler CM (2009) Challenges in evaluating surgical innovation. Lancet 374(9695):1097-1104

14. Foks KA, Cnossen MC, Dippel DWJ, Maas A, Menon D, van der Naalt J, Steyerberg EW, Lingsma H, Polinder S (2017) Management of mild traumatic brain injury at the emergency department and hospital admission in Europe: a survey of 71 neurotrauma centers participating in the CENTER-TBI study. J Neurotrauma. https://doi.org/10.1089/neu.2016.4919

15. Ghajar J, Carney N (2013) Intracranial-pressure monitoring in traumatic brain injury. N Engl J Med 368(18): 1749

16. Gregson BA, Mendelow AD (2003) International variations in surgical practice for spontaneous intracerebral hemorrhage. Stroke 34(11):2593-2597

17. Hesdorffer DC, Ghajar J (2007) Marked improvement in adherence to traumatic brain injury guidelines in United States trauma centers. The Journal of Trauma: Injury, Infection, and Critical Care 63(4): 841-848

18. Huijben JA, van der Jagt M, Cnossen MC et al (2017) Variation in blood transfusion and coagulation management in traumatic brain injury at the intensive care unit: a survey in 66 neurotrauma centers participating in the collaborative European NeuroTrauma Effectiveness Research in Traumatic Brain Injury (CENTER-TBI) study. Journal of Neurotrauma neu 2017:5194

19. Hutchinson PJ, Kolias AG, Timofeev IS et al (2016) Trial of decompressive craniectomy for traumatic intracranial hypertension. $\mathrm{N}$ Engl J Med 375(12):1119-1130

20. Kahle KT, Duhaime A-C (2013) Intracranial-pressure monitoring in traumatic brain injury. N Engl J Med 368(18):1750

21. Kolias AG, Scotton WJ, Belli A et al (2013) Surgical management of acute subdural haematomas: current practice patterns in the United Kingdom and the Republic of Ireland. Br J Neurosurg 27(3):330-333

22. Li LM, Kolias AG, Guilfoyle MR, Timofeev I, Corteen EA, Pickard JD, Menon DK, Kirkpatrick PJ, Hutchinson PJ (2012)
Outcome following evacuation of acute subdural haematomas: a comparison of craniotomy with decompressive craniectomy. Acta Neurochir 154(9):1555-1561

23. Lingsma HF, Roozenbeek B, Li B, Lu J, Weir J, Butcher I, Marmarou A, Murray GD, Maas AIR, Steyerberg EW (2011) Large between-center differences in outcome after moderate and severe traumatic brain injury in the international mission on prognosis and clinical trial design in Traumatic Brain Injury (IMPACT) study. Neurosurgery 68(3):601-608

24. Maas AIR, Menon DK, Lingsma HF, Pineda JA, Sandel ME, Manley GT (2012) Re-orientation of clinical research in traumatic brain injury: report of an international workshop on comparative effectiveness research. J Neurotrauma 29(1):32-46

25. Maas AIR, Menon DK, Steyerberg EW, Citerio G, Lecky F, Manley GT, Hill S, Legrand V, Sorgner A (2015) Collaborative European NeuroTrauma effectiveness research in traumatic brain injury (CENTER-TBI): a prospective longitudinal observational study. Neurosurgery 76(1):67-80

26. Maas AIR, Stocchetti N, Bullock R (2008) Moderate and severe traumatic brain injury in adults. The Lancet Neurology 7(8):728 741

27. Macefield RC, Boulind CE, Blazeby JM (2014) Selecting and measuring optimal outcomes for randomised controlled trials in surgery. Langenbeck's Arch Surg 399(3):263-272

28. Mattei TA (2013) Intracranial pressure monitoring in severe traumatic brain injury: who is still bold enough to keep sinning against the level I evidence? World Neurosurg 79(5-6):602-604

29. Mendelow AD, Gregson BA, Rowan EN, Francis R, McColl E, McNamee P, Chambers I, Unterberg AW, Boyers D, Mitchell P (2015) Early surgery versus initial conservative treatment in patients with traumatic intracerebral haemorrhage [STITCH (trauma)]: the first randomised trial. J Neurotrauma. https://doi.org/10. 1089/neu.2014.3644

30. Murray GD, Teasdale GM, Braakman R et al (1999) The European brain injury consortium survey of head injuries. Acta Neurochir 141(3):223-236

31. Rayan N, Barnes S, Fleming N, Kudyakov R, Ballard D, Gentilello LM, Shafi S (2012) Barriers to compliance with evidence-based care in trauma. J Trauma Acute Care Surg 72(3):585-92- discussion 592-3

32. Servadei F, Compagnone C, Sahuquillo J (2007) The role of surgery in traumatic brain injury. Curr Opin Crit Care 13(2):163-168

33. Timmons SD, Toms SA (2012) Comparative effectiveness research in neurotrauma. Neurosurg Focus 33(1):E3

34. van Essen TA, de Ruiter GCW, Kho KH, Peul WC (2016) Neurosurgical treatment variation of traumatic brain injury: evaluation of acute subdural hematoma management in Belgium and the Netherlands. J Neurotrauma doi: https://doi.org/10.1089/neu.2016. 4495

35. Van Essen TA, Dijkman M, Cnossen MC, Moudrous W, Ardon H, Schoonman GG, Steyerberg EW, Peul W, Lingsma H, de Ruiter GCW (2018) Comparative effectiveness of surgery for traumatic acute subdural hematoma in an aging population. J Neurotrauma. https://doi.org/10.1089/neu.2018.5869 